\title{
Evaluación de posibles medidas para reducir las emisiones de fuentes móviles en Costa Rica, 2010-20I5
}

\author{
Assessment of possible strategies to reduce mobile \\ sources emissions in Costa Rica, 2010-2015 projection
}

Jorge Herrera-Murillo'

José Félix Rojas-Marín ${ }^{2}$

Susana Rodríguez-Román ${ }^{3}$

Fecha de recepción: 06 de mayo del 2013

Fecha de aprobación: 19 de julio del 2013

Herrera-Murillo, j; Rojas-Marín, J; Rodríguez-

Román, S. Evaluación de posibles medidas para reducir las emisiones de fuentes móviles en Costa Rica, 2010-2015. Tecnología en Marcha. Vol. 27, N I. Pág 23-37

Laboratorio de Análisis Ambiental, Escuela de Ciencias Ambientales, Universidad Nacional. Apartado 86-3000 Heredia, Costa Rica. Correo electrónico: jorge.herrera.murillo@una.cr.

2 Laboratorio de Análisis Ambiental, Escuela de Ciencias Ambientales, Universidad Nacional. Apartado 86-3000 Heredia, Costa Rica. Correo electrónico: jose.rojas.marin@una.cr.

3 Escuela de Química, Universidad de Costa Rica, Ciudad Universitaria Rodrigo Facio, San José. Costa Rica. Correo electrónico: susana.rodriguez@ucr.ac.cr. 


\section{Palabras clave}

Fuentes móviles; emisiones; reducción; Costa Rica; contaminación del aire.

\section{Resumen}

Se evaluó el impacto de las posibles medidas para reducir las emisiones generadas por fuentes móviles en Costa Rica para ser ejecutadas en el periodo 2010-2015. La estimación de las emisiones se realizó utilizando los factores de emisión obtenidos del modelo Mobile 6 y los datos de actividad distribuidos por tipo de vehículo y combustible. El estudio determinó que la sustitución del 50\% de las unidades de transporte público por vehículos más eficientes resulta ser la medida más eficaz para contrarrestar el incremento de las emisiones de NOx y GOT, cercanas al I4,3\% y $11,7 \%$ anual respectivamente.

\section{Keywords}

Mobile sources emissions ; Costa Rica ; emissions reduction ; air pollution.

\begin{abstract}
The impacts of the possible strategies to reduce the emissions from mobile sources in Costa Rica were evaluated for the 2010-20I 5 period. The total emissions were estimated using emission factors obtained from Mobile 6 model and activity data like fuel and vehicle type distribution. This study found that 50\% substitution of public transport vehicles was the most effective measure to lower the anual rate increase for $\mathrm{NOx}$ and Total Organic Gases (TOG). Both around 14,3\% and I I,7\% anually, respectively.
\end{abstract}

\section{Introducción}

Los vehículos de transporte son las principales fuentes de emisión de contaminantes del aire, especialmente en las zonas urbanas, así como el origen de la mayoría de los problemas de externalidades ambientales (Button, 1993). Las emisiones de contaminantes por las fuentes móviles carreteras están directamente relacionadas con las necesidades de movilidad particular y/o comercial de una región, las que a su vez son proporcionales al crecimiento poblacional y al comportamiento de la economía predominante en la zona (Parks y Hrunka, 2000).

El enfoque general que se utiliza para el cálculo de las emisiones generadas por este tipo de fuente contempla dos etapas. En primera instancia, se debe desarrollar un conjunto de factores de emisión que representen la tasa de emisión por unidad de actividad. Estos factores pueden definirse bajo condiciones controladas de laboratorio para ciclos de conducción predeterminados (Adler, 1997), mediante pruebas de manejo en tiempo real sobre carreteras, utilizando instrumentos a bordo que miden las emisiones generadas (Journard et al., 1995; Kelly y
Groblicki, I993; Bishop y Stedman, 1990) o por el uso de software tales como MOBILE 6, IVE, etc.

La segunda etapa implica la determinación de una estimación de los vehículos y/o la actividad de tráfico. Estos datos de actividad se pueden derivar tanto de modelos como de conteos o encuestas de tráfico (Cardelino, 1998). Al igual que con las mediciones de emisiones a bordo, los datos de las encuestas son más deseables ya que proveen información sobre los patrones reales de transporte en los distintos tipos de caminos.

$\mathrm{Si}$ se considera que la flota vehicular registrada en Costa Rica hasta enero de 2010 corresponde a I 079 33| unidades (I vehículo por cada 4,2 habitantes), de las cuales un 63,8\% son vehículos particulares y tan solo un 2,8\% está destinado al transporte público (figura I), se hace necesario planificar adecuadamente las políticas públicas que deberán ser implementadas con el fin de controlar las emisiones vehiculares y así garantizar el cumplimiento, tanto actual como futuro, de las normas de calidad del aire, sobre todo si se considera que: 


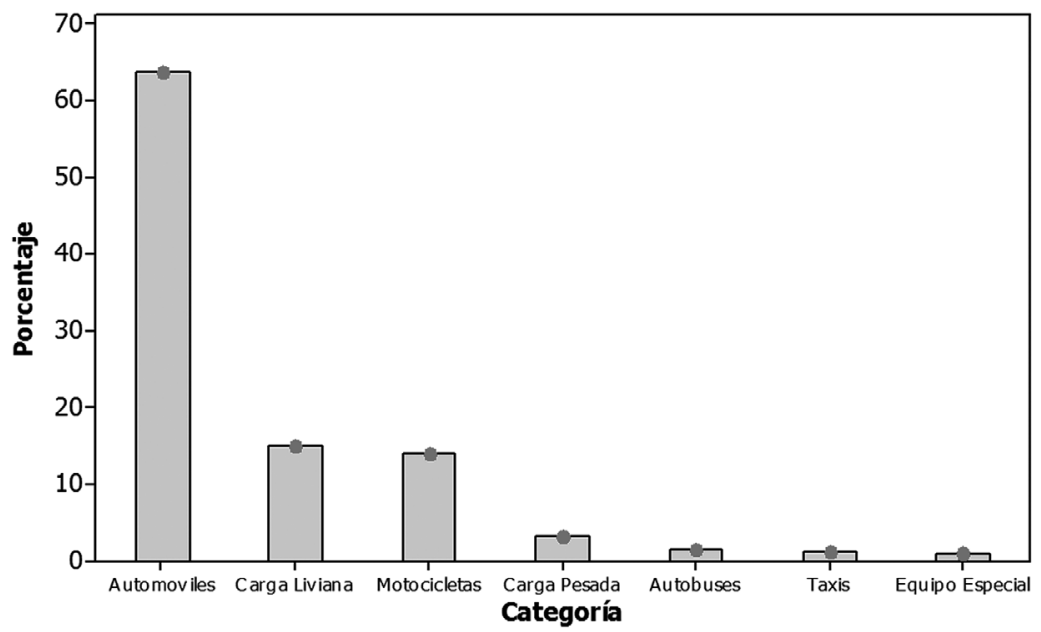

Figura I. Composición porcentual por categoría de la flota vehicular de Costa Rica hasta enero 2010.

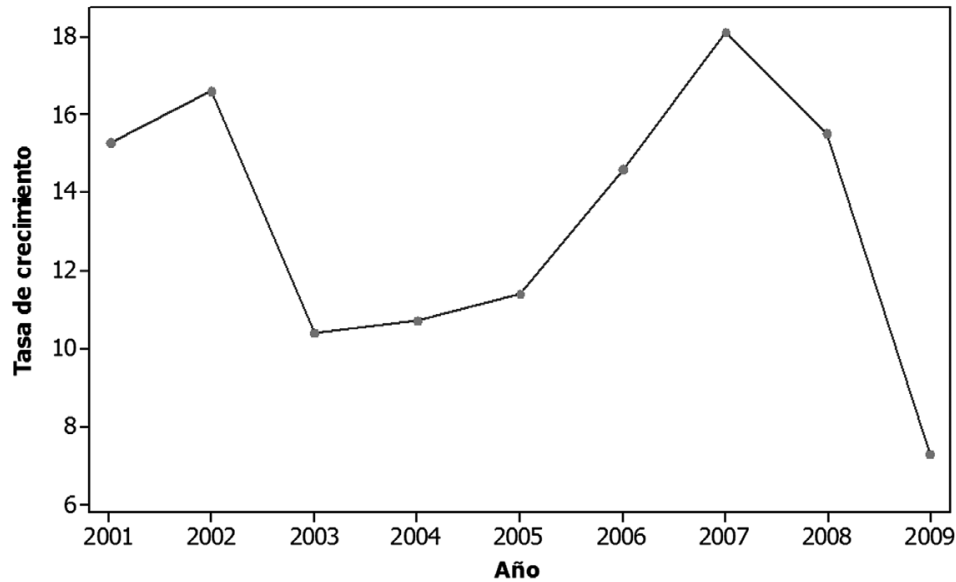

Figura 2. Comportamiento de la tasa de crecimiento anual de la flota vehicular de Costa Rica (serie de tiempo 200 I2009).

- Del total de unidades que integran la flota, $77,7 \%$ utiliza gasolina, mientras que 22,2\% emplean diesel como combustible. De los vehículos a gasolina, el 20,8\% son modelos 1990 y anteriores, que no cuentan con sistemas de control de emisiones; aproximadamente el 10,6\% son modelos 199|-1992, vehículos que ya integran convertidores catalíticos de dos vías y permiten disminuir las emisiones de hidrocarburos y monóxido de carbono. Los vehículos que corresponden a modelos 1993 y posteriores (68,7\% de la flota a gasolina) ya cuentan con convertidores catalíticos de tres vías y otros dispositivos anticontaminantes para reducir las emisiones de hidrocarburos, monóxido de carbono y óxidos de nitrógeno. En lo que se refiere a las unidades que utilizan diesel, alrededor del 28,1\% son unidades año modelo 1993 y anteriores (sin control de emisiones). Cerca del 71,9\% son vehículos que ya traen mejoras tecnológicas para cumplir con los estándares de emisión, tales como EPA 94 y EPA 98 respectivamente (INS, 2010).

- La tasa de crecimiento de la flota vehicular se ha mantenido cercana al I3,3\% en el período 200 I 2009 (figura 2). De las incorporaciones anuales a la flota, aproximadamente un promedio de 
58,8\% corresponde a vehículos usados, de los cuales 65\% tienen una edad de hasta 5 años, $13 \%$ de 5 a 10 años, $16 \%$ de 10 a 15 años y $7 \%$ de más de 15 años.

- El tránsito privado ha venido creciendo a un ritmo del $8 \%$ anual y la infraestructura vial no ha sido desarrollada proporcionalmente. Esto está provocando la saturación del sistema vial con una fuerte congestión de tránsito, altas emisiones contaminantes sobre el ambiente, deseconomías por desperdicio de hidrocarburos, altos costos de operación de los vehículos y del tiempo de los usuarios y otros efectos indeseables de alto impacto (Flores, 2008).

En el presente artículo se evalúan algunas alternativas, en términos de reducción, que pueden ser implementadas con el fin de controlar las emisiones generadas por fuentes móviles carreteras en Costa Rica.

\section{Metodología}

\section{Fuentes de información}

Para realizar este análisis se partió de la información de la flota vehicular de Costa Rica registrada hasta enero del año 2010, suministrada por el Registro Nacional, en la que se indica: categoría del vehículo, tipo de combustible, año modelo, peso, etc. En el cuadro | se muestran otras fuentes de información utilizadas en este artículo.

Cálculo de las emisiones para los escenarios propuestos

Para estimar el potencial de emisiones del parque vehicular para cada uno de los escenarios propuestos, se obtuvieron los factores de emisión para los distintos contaminantes criterio a partir del software Mobile 6, que es un modelo computacional desarrollado por la Agencia de Protección Ambiental de Estados Unidos (EPA, por sus siglas en inglés) y modificado de acuerdo con las condiciones imperantes en Costa Rica. Los factores de emisión obtenidos del modelo se estimaron por clase de vehículo, tipo de combustible y año modelo.

Los datos de actividad se tomaron de la encuesta del recorrido medio anual de los vehículos en circulación en el país elaborada por la Dirección Sectorial de Energía (cuadro 2). En este estudio se utilizaron cuatro métodos de cálculo para el recorrido anual en kilómetros:

Método I: Estimación según "sticker" y odómetro. Consiste en hacer un cálculo del recorrido tomando en cuenta la fecha del último cambio de aceite al automotor y la marca del odómetro al próximo cambio de aceite.

Método 2: Estimación según declaración de la marca del odómetro cuando se compró el automotor y el día de la entrevista. En este método de cálculo se consulta si el vehículo era nuevo o usado y si el conductor sabía la marca del odómetro el día de la compra.

Método 3: Estimación según declaración del rendimiento del vehículo en kilómetros por galón. En este método se le pregunta al conductor por el rendimiento en kilómetros por galón en forma general, en la ciudad y en carretera.

Método 4: Estimación según llamada posterior al conductor. Este método consiste en solicitar al conductor del automotor la posibilidad de llamarle varios días después para que declarara la marca del odómetro ese día; entonces, con la diferencia entre la marca del odómetro el día de la entrevista y la marca del odómetro el día de la llamada dividido por el número de días transcurridos entre ambas fechas se calcula un recorrido diario y la estimación anual respectiva.

Para obtener un dato único de recorrido anual en kilómetros para cada automotor, se calculó un promedio entre los datos que se estimaron según los cuatro métodos. Con los datos del recorrido diario, los días que circularon los vehículos durante el año del escenario y el número de vehículos de acuerdo a la distribución por año modelo, se obtuvieron los kilómetros recorridos (KRV) por tipo de vehículo y año modelo, a partir de la ecuación:

$$
K R V i j=(K D j)(N V i j)(D A i)(I)
$$

donde:

$$
\begin{aligned}
\text { KRVij }= & \text { Kilómetros recorridos por el tipo de vehí- } \\
& \text { culo i, del año modelo j [km/año]. } \\
\text { KDj = } & \text { Kilómetros recorridos al día por el tipo de } \\
& \text { vehículo } \mathrm{i}[\mathrm{km} / \mathrm{d} \text { áa]. }
\end{aligned}
$$


Cuadro I. Fuentes de información utilizadas en el estudio.

\begin{tabular}{|l|l|l|}
\hline \multicolumn{1}{|c|}{ Tipo de información } & \multicolumn{1}{|c|}{ Características } & \multicolumn{1}{c|}{ Fuente } \\
\hline $\begin{array}{l}\text { Características fisicoquímicas } \\
\text { de los combustibles }\end{array}$ & $\begin{array}{l}\text { Diesel: } \\
\text {-Contenido de azufre (2003-2009) } \\
\text { Gasolina: } \\
\text {-Contenido de azufre (2003-2009) } \\
\text {-Presión de Vapor Reid (2003-2009) } \\
\text {-Contenido de oxigenados (2003-2009) }\end{array}$ & $\begin{array}{l}\text { Refinadora Costarricense de Petróleo } \\
\text { (RECOPE) }\end{array}$ \\
\hline Datos meteorológicos & $\begin{array}{l}\text {-Máximos y mínimos diarios de temperatura (2003- } \\
\text { 2009) }\end{array}$ & $\begin{array}{l}\text { Instituto Meteorológico Nacional } \\
\text { (IMN) }\end{array}$ \\
\hline Datos económicos & $\begin{array}{l}\text {-Tasa de inflación (1992-2009) } \\
\text {-Producto Interno Bruto nacional (1992-2009) }\end{array}$ & $\begin{array}{l}\text { Ministerio de Planificación y Política } \\
\text { Económica (MIDEPLAN), } \\
\text { Banco Central de Costa Rica (BCCR) }\end{array}$ \\
\hline $\begin{array}{l}\text { Datos de población } \\
\text { Datos de importaciones de } \\
\text { vehículos }\end{array}$ & $\begin{array}{l}\text {-Importaciones de vehículos nuevos y usados (2009- } \\
\text { 200I) por año modelo }\end{array}$ & $\begin{array}{l}\text { Instituto Nacional de Estadísticas y } \\
\text { Censos (INEC) }\end{array}$ \\
\hline $\begin{array}{l}\text { Datos de kilómetros totales } \\
\text { recorridos por vehículos en } \\
\text { Costa Rica }\end{array}$ & $\begin{array}{l}\text {-Kilómetros anuales recorridos por la flota vehicular } \\
\text { por categoría de vehículo y tipo de combustible. }\end{array}$ & $\begin{array}{l}\text { Ministerio de Hacienda } \\
\text { Dirección Sectorial de Energía, } \\
\text { Ministerio de Ambiente, Energía y } \\
\text { Telecomunicaciones }\end{array}$ \\
\hline
\end{tabular}

Cuadro 2. Datos de actividad de la flota vehicular que circula en el área metropolitana de Costa Rica.

\begin{tabular}{|l|c|}
\hline \multicolumn{1}{|c|}{ Categoría de vehículo } & Kilómetros promedio recorridos en un año \\
\hline Automóvil particular & 20768 \\
\hline Carga liviana & 27990 \\
\hline Carga pesada & 38934 \\
\hline Taxis & 86109 \\
\hline Buses & 43417 \\
\hline Motos & 16091 \\
\hline
\end{tabular}

NVij = Número de vehículos del tipo i, del año modelo j.

DAi = Días al año que circulan los vehículos del tipo i [días/año].

A partir de los kilómetros recorridos (KRV) y los factores de emisión por tipo de vehículo, combustible y año modelo, se obtuvieron las emisiones de cada contaminante, de acuerdo a la siguiente ecuación:

$$
E_{i j k}=\left(K R V_{i j}\right)\left(F_{i j k}\right) /(1000000)(2)
$$

donde:

$\mathrm{E}_{\mathrm{ijk}} \quad=$ Emisión del tipo de vehículo $\mathrm{i}$, año modelo j, del contaminante $k$ [ton/año]

$\mathrm{KRV}_{\mathrm{ij}}=$ Kilómetros recorridos por el tipo de vehículo i, año modelo j [km/año]

$\mathrm{FE}_{\mathrm{ijk}} \quad=$ Factor de emisión del tipo de vehículo $\mathrm{i}$, año modelo j, del contaminante $\mathrm{k}[\mathrm{g} /$ $\mathrm{km}]$

$1000000=$ Factor de conversión de gramos a toneladas. 


\section{Proyección de la flota vehicular}

La modelación del transporte es un proceso de desarrollo de modelos matemáticos aplicables al pronóstico de las características del sistema de transporte de una región, tales como: el volumen de las flotas vehiculares, la edad del parque vehicular, el nivel de saturación (autos/I00 habitantes, \%), viajes y tráfico, entre otras.

Algunas de las variables comúnmente empleadas para modelar el transporte son la densidad de población, la tasa de empleo, el Producto Interno Bruto (PIB), el ingreso per cápita y la inflación. El modelo de Quarmby y Bates (1970), por ejemplo, emplea dos variables independientes: el ingreso y la densidad residencial, para modelar las flotas de autos particulares, aunque reconoce la existencia de otros factores de interés como el tamaño de la localidad y el precio de los vehículos (Ortizar et al., 1994). Las relaciones básicas del modelo de Quarmby son:

$$
\begin{aligned}
& \frac{P_{0}}{1-P_{0}}=\alpha_{0} I^{-b_{0}} D^{C_{0}} \\
& \frac{P_{2}}{P_{1}}=a_{1} e^{\left(b_{1} I\right)} D^{-C_{1}} \\
& P_{0}+P_{1}+P_{2}=1
\end{aligned}
$$

donde:

$$
\begin{aligned}
\mathrm{Pi}= & \text { Probabilidad de que una residencia posea } \mathrm{i} \\
& \text { vehículos } \\
\mathrm{I}= & \text { Ingreso anual familiar } \\
\mathrm{D}= & \text { número de residentes por acre }
\end{aligned}
$$
ai, bi y ci son los parámetros a estimar $\alpha_{0}=$ Índice de saturación, fracción

Sin embargo, la variable del ingreso per cápita no refleja la pérdida de poder adquisitivo del ingreso, que suele ser muy significativa en países como Costa Rica. El modelo de Quarmby y Bates considera el nivel de saturación la cual genera el efecto de límite del crecimiento del parque vehicular que se observa en países desarrollados altamente motorizados como Estados Unidos de Norteamérica. Estas estimaciones consideran tres características del parque vehicular a modelar: número de vehículos, distribución de los vehículos por tamaño y distribución de los vehículos por tipo de combustible.
Para el presente trabajo se tomaron como base los estudios realizados por el Instituto Mexicano del Petróleo, los cuales han supuesto que el crecimiento de los vehículos de uso comercial es proporcional al crecimiento de la relación PIB/persona (Gasca, 2005), suponiendo el crecimiento del PIB como una constante y tomando las proyecciones de crecimiento poblacional del Instituto Nacional de Estadísticas y Censos de Costa Rica.

\section{Escenarios evaluados}

Las acciones consideradas en el presente trabajo como posibles iniciativas para la reducción y el control de emisiones de fuentes móviles en Costa Rica se indican a continuación:

- Acción I: Reducir el contenido de azufre en el diesel de 400 a 100 ppm para el año 2015.

- Acción 2: Reemplazo del 100\% de unidades de autobuses y taxis con edades superiores a los 10 años para el periodo 2010-20I5.

- Acción 3: Aplicación de medidas de restricción vehicular de un día por semana de acuerdo con el número de placa del vehículo.

- Acción 4: Prohibición de importación de vehículos usados con más de cinco años de antigüedad a partir del año 2010.

- Acción 5: Incentivar el uso de gas licuado de petróleo o unidades de alto rendimiento en el $50 \%$ de las unidades de transporte público para el año 2015.

\section{Resultados}

\section{Evolución de la flota vehicular 2010-20।5}

Con el fin de elaborar un modelo para pronosticar el crecimiento de la flota vehicular al año 2015, se estimó el comportamiento del PIB del país a partir de los datos de la serie histórica 1992-2009, obtenida del Ministerio de Planificación y Política Económica y los pronósticos publicados por el Banco Central de Costa Rica para los años 2010 y 20II. En el cuadro 3 se presentan los datos empleados para el pronóstico del PIB. Para mejorar el pronóstico se utilizó la inflación como variable de ajuste de la jornada financiera.

Se aplicaron II modelos de series de tiempo para identificar el que mejor describe el comportamiento 
Cuadro 3. Datos empleados para el pronóstico del PIB.

\begin{tabular}{|c|c|c|}
\hline Año & Inflación (\%) & $\begin{array}{c}\text { Variación del PIB } \\
\text { (fracción) }\end{array}$ \\
\hline 1992 & 16,97 & 0,0915 \\
\hline 1993 & 9,04 & 0,0741 \\
\hline 1994 & 19,86 & 0,0473 \\
\hline 1995 & 22,57 & 0,0392 \\
\hline 1996 & 13,89 & 0,0089 \\
\hline 1997 & I I,20 & 0,0558 \\
\hline 1998 & 12,36 & 0,0840 \\
\hline 1999 & 10,11 & 0,0822 \\
\hline 2000 & 10,25 & 0,0180 \\
\hline 2001 & 10,96 & 0,0108 \\
\hline 2002 & 9,68 & 0,0290 \\
\hline 2003 & 9,87 & 0,0640 \\
\hline 2004 & 13,13 & 0,0426 \\
\hline 2005 & |4,07 & 0,0589 \\
\hline 2006 & 9,43 & 0,0878 \\
\hline 2007 & $|0,8|$ & 0,0795 \\
\hline 2008 & 13,9 & 0,0282 \\
\hline 2009 & 4,05 & $-0,0128$ \\
\hline 2010 & 8,44 & 0,0400 \\
\hline
\end{tabular}

de la variación del PIB: (a) recorrido aleatorio con tendencia, (b) media constante, (c) tendencia lineal $=2,837-0,00139 t$, (d) suavizado exponencial simple con $\alpha=0,0328$, (e) suavizado exponencial lineal de Brown con $\alpha=0,0 \mid 46$, (f) suavizado exponencial lineal de Holt con $\alpha=0,2519$ y $\beta=0,1424$, (g) ARIMA $(2,0,1)$ con constante, (h) ARIMA $(2,0,2)$ con constante, (i) ARIMA (2, I, I), (j) ARIMA $(2,0,0)$ con constante, (k) ARIMA $(2,1,1)$ con constante. Para identificar el modelo apropiado se utilizaron métodos estadísticos de medición de la precisión del pronóstico.

En el análisis estadístico de la precisión del pronóstico se calcularon los estadísticos del error:

$$
\begin{gathered}
E M=\frac{\sum_{t=1}^{n} e_{t}}{n} \\
E M A=\frac{\sum_{t=1}^{n}\left|e_{t}\right|}{n} \\
R E C M=\sqrt{\frac{\sum_{t=1}^{n} e_{t}^{2}}{n}}
\end{gathered}
$$

donde:

$E M=$ Error medio.

$E M A=$ Error medio absoluto.

RECM = Raíz del error cuadrado medio.

et $=$ error del pronóstico al periodo de tiempo t.

$n=$ número de observaciones en el periodo de observación.

De manera que el mejor modelo será aquel que presente el valor más cercano a cero del EM y el valor más pequeño de los estadísticos EMA y RECM. El cuadro 4 resume los valores de estos estadísticos para los modelos probados, en donde se observa que el modelo $(G)$ presenta el menor valor del RECM y del EMA. En forma adicional, el cuadro 4 contiene los resultados de las pruebas, en donde se identificó que el error no es significativo a un nivel de $95 \%$ de confianza para los modelos de $(G)$ a $(K)$. Se puede identificar en la figura 3 que el modelo $(G)$ se ajusta a los datos y presenta una sensibilidad adecuada a los cambios bruscos del PIB. En el presente estudio, la sensibilidad y ajuste del modelo a los datos tiene alta relevancia, debido a que la variable endógena Variación PIB se empleará como variable exógena o explicatoria para las proyecciones de las emisiones contaminantes de escenarios a corto y mediano plazo. Por lo tanto, se seleccionó el modelo (G) de ARIMA $(2,0,1)$ con constante como el más apropiado para pronosticar las variaciones del PIB.

En el cuadro 5 se muestran los resultados del pronóstico del cambio en el PIB nacional de Costa Rica para el período 2010-20I5, así como los intervalos de confianza al 95\%. Se puede observar que los intervalos se hacen más amplios conforme el pronóstico se aleja en el tiempo.

Si se plantea la hipótesis de que el crecimiento del parque vehicular en Costa Rica dependerá del volumen de ventas automotrices, las cuales están directamente relacionadas con la capacidad 
Cuadro 4. Estadísticos de error para los modelos probados para el pronóstico del PIB.

\begin{tabular}{|c|c|c|c|c|c|c|c|c|}
\hline Modelo & RECM & EMA & EM & RUNS & RUNM & AUTO & MEAN & VAR \\
\hline (A) & 0,03277 & 0,02751 & $-7,71 \times 10^{-19}$ & OK & OK & OK & OK & OK \\
\hline (B) & 0,02945 & 0,02503 & $-5,48 \times 10^{-18}$ & $*$ & OK & OK & OK & OK \\
\hline$($ C $)$ & 0,02922 & 0,02410 & $-5,37 \times 10^{-17}$ & $*$ & OK & OK & OK & OK \\
\hline (D) & 0,03009 & 0,02545 & 0,00202 & $*$ & OK & OK & OK & OK \\
\hline (E) & 0,03012 & 0,02549 & 0,00212 & $*$ & OK & OK & OK & OK \\
\hline$(F)$ & 0,03398 & 0,02882 & 0,00293 & $*$ & OK & OK & OK & OK \\
\hline (G) & 0,01920 & 0,01394 & $-0,00066$ & OK & OK & OK & OK & OK \\
\hline (H) & 0,01827 & 0,01264 & 0,00136 & OK & OK & OK & OK & OK \\
\hline$($ I) & 0,02119 & 0,01695 & 0,00018 & OK & OK & OK & OK & OK \\
\hline (J) & 0,02134 & 0,01710 & 0,00050 & OK & OK & OK & OK & OK \\
\hline (K) & 0,02068 & 0,01555 & 0,00060 & OK & OK & OK & OK & OK \\
\hline
\end{tabular}

RUNS = Test para excesivas ejecuciones arriba y abajo

RUNM = Test para excesivas ejecuciones por encima y por debajo de la mediana

AUTO $=$ Test de Box-Pierce para excesivas autocorrelaciones

MEAN = Test para la diferencia en la media de la $I^{\mathrm{a}}$ mitad a la $2^{\mathrm{a}}$ mitad

$\mathrm{VAR}=$ Test para la diferencia en la varianza en la $\mathrm{I}^{\mathrm{a}}$ mitad a la $2^{\mathrm{a}}$ mitad

$\mathrm{OK}=$ no significativa $\left(\mathrm{P}^{3} 0,05\right)$

* = marginalmente significativa $(0,01<p \in 0,05)$

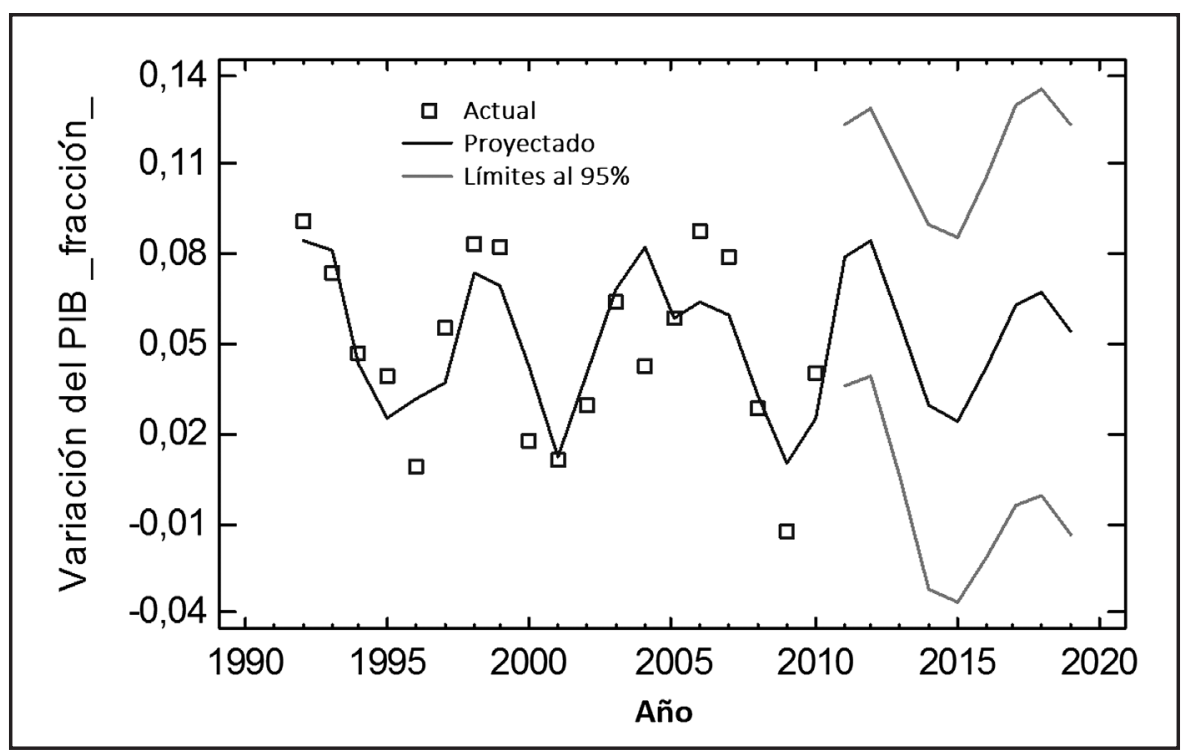

Figura 3. Proyección de la tasa de variación anual del PIB de Costa Rica (modelo ARIMA $(2,0,1)$ ). 
Cuadro 5. Valores pronosticados del PIB con intervalos al 95\% de confianza.

\begin{tabular}{|c|c|c|c|}
\hline Período & Predicción & L. inferior & L. superior \\
\hline 2011 & 0,0736 & 0,0357 & 0,1103 \\
\hline 2012 & 0,08264 & 0,0448 & 0,1205 \\
\hline 2013 & 0,06544 & 0,0204 & 0,1105 \\
\hline 2014 & 0,03866 & $-0,0165$ & 0,0938 \\
\hline 2015 & 0,0239 & $-0,0331$ & 0,0808 \\
\hline
\end{tabular}

económica de la población (cantidad de dinero que posee la población asociado al valor que ese dinero tiene en el mercado), se puede hacer un análisis de regresión múltiple que asocie las ventas nacionales al PIB, la tasa de inflación y la población del país.

En el cuadro 6 se resumen los estadísticos correspondientes al ajuste de los diferentes modelos de regresión y en ella se puede observar que individualmente, la variable $\mathrm{B}$ (tasa de inflación) es la que tiene el valor más alto de ECM, el valor de $\mathrm{R}^{2}$ ajustado y coeficiente de Durbin-Watson más bajo.

Cuadro 6. Resultados del ajuste del modelo de regresión lineal para las ventas de vehículos.

\begin{tabular}{|c|c|c|c|}
\hline EMA & $\mathrm{R}^{2}$ ajustado (\%) & $\begin{array}{c}\text { Estadístico } \\
\text { Durbin Watson }\end{array}$ & Variables incluidas \\
\hline 16002,2 & 57,5 & 1,5698 & $A$ \\
\hline 26746,4 & 0,0 & 0,6286 & B \\
\hline 19484,3 & 38,4 & 1,4744 & C \\
\hline 14759,4 & 63,1 & ।,3077 & $A, B$ \\
\hline 7401,7 & 88,7 & 2,9964 & $A, C$ \\
\hline 24865,8 & 43,2 & 1,2245 & $\mathrm{~B}, \mathrm{C}$ \\
\hline 3066,98 & 99,4 & 2,9499 & $A, B, C$ \\
\hline
\end{tabular}

$A=$ Producto Interno Bruto, B= Tasa de Inflación, $C=$ Población.

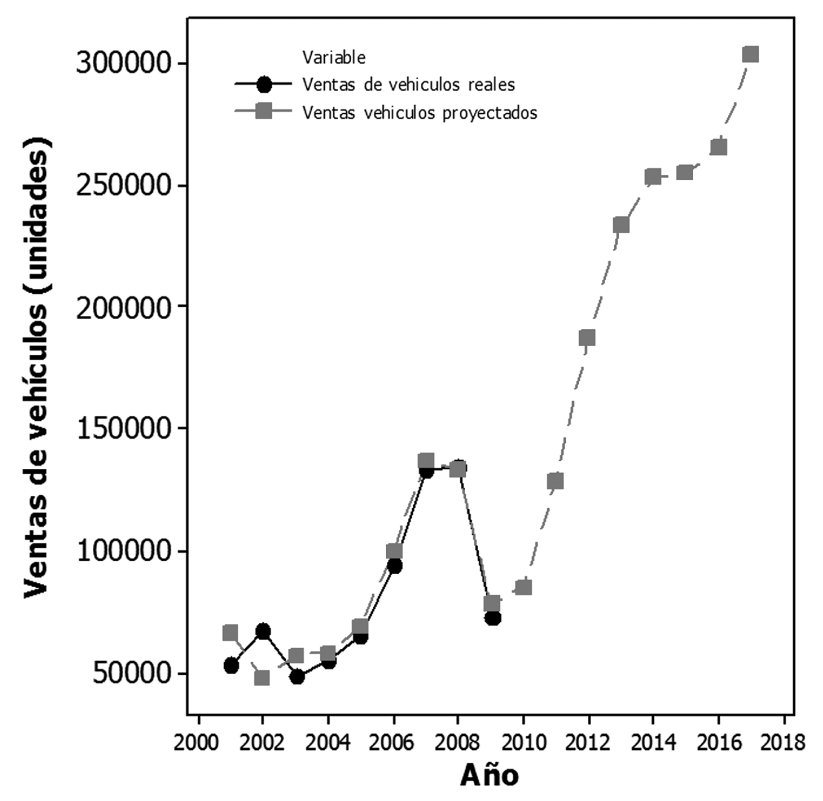

Figura 4. Proyección de las ventas anuales de vehículos en Costa Rica (2010-2015).
Estas condiciones significan que la tasa de inflación es la variable que menos explica las ventas nacionales de autos. El modelo de regresión que incluye las variables PIB, inflación y población es la que cumple con los criterios de mejor combinación. Esta combinación genera valores de $\mathrm{R}^{2}$ ajustado más altos y los menores valores de ECM. El modelo de regresión múltiple resultante está dado por la ecuación 5 y la figura 4 muestra la evolución y el modelo ajustado de las ventas nacionales.

Ventas nacionales $=\mid 543480+0,459$ PIB $-0,537$ Población + 1232,3 Tasa Inflación (5)

Los modelos de regresión de las flotas vehiculares permiten proyectar el volumen total de cada tipo de vehículo. Para complementar la proyección del parque vehicular se requiere conocer la distribución por edad de la flota. Para pronosticar esta información se deben conocer las curvas de retiro, 
que describen la velocidad a la cual se retiran las unidades. Estas curvas son específicas para cada tipo de vehículo dado el diferente uso que se les da y la tecnología que incorporan. Las curvas de retiro incluso pueden cambiar con el año modelo para vehículos del mismo tipo, debido al diseño de estos.

Utilizando los datos de distribución de la flota vehicular por categorías para el período 200I-2010 se corrieron pruebas de selección de modelos de regresión, empleando las tres variables explicatorias: PIB, inflación y población. Estos modelos describen el crecimiento del parque de cada tipo de vehículo y se exponen en el cuadro 7. El cuadro 8 contiene los valores proyectados para la flota vehicular por categoría de vehículo para el periodo 20 I -20 I7.

Empleando las bases de datos de la flota vehicular del 200 I al 2010 por año modelo y tipo de vehículo para Costa Rica se identificaron las curvas de retiro características del parque vehicular. Al analizar estas curvas se propusieron los modelos para determinar el número de vehículos de un año modelo dado para el año calendario que se desee. Las curvas de retiro se definieron por el modelo generalizado:

flota $_{i, j, t}=f\left(\right.$ flota $_{i, j, t-1}$, edad $\left._{i, j t}, e r_{i}\right)$

donde:

Flota i,j,t = Número de vehículos de tipo i del año modelo $j$ en el año calendario $t$

Flota i,j,t- I = Número de vehículos de tipo i del año modelo $j$ en el año calendario $t-1$

Edad i,j,t = Edad de los vehículos de tipo i del año modelo $j$ al año calendario $t$

eri $\quad=$ Edad típica de retiro de los vehículos del tipo $i$

Edad $=$ año calendario - año modelo $=t-j$

Por lo que se puede observar en la ecuación (6), se está proponiendo una ecuación para cada tipo de

Cuadro 7. Modelos de regresión múltiple para las distintas categorías de vehículos en la flota.

\begin{tabular}{|c|c|c|c|}
\hline Categoría & Modelo de regresión & EMA & $\mathrm{R}^{2}$ ajustado \\
\hline Aut. particulares & -3689350 - 0,1 68PIB + I,0424Población-5 I 62,2Inflación & 19153,8 & 96,37 \\
\hline Taxis & -73292 - 0,00334PIB + 0,0207Población - 102,6Inflación & 380,5 & 96,88 \\
\hline Autobuses & -89420 - 0,00407PIB + 0,0253Población - I25,09Inflación & 464,3 & 96,81 \\
\hline Motocicletas & -807| I I - 0,0367PIB + 0,228Población - I I29,35 Inflación & 4190,3 & 97,03 \\
\hline Carga liviana & -87|793 - 0,0397PIB + 0,246Población - 1219,84 Inflación & 4526,1 & 96,55 \\
\hline Carga pesada & -19|665 - 0,00873PIB + 0,054|Población - 268,2 Inflación & 995,2 & 97,58 \\
\hline
\end{tabular}

Cuadro 8. Proyección de las flotas vehiculares de 2011 a 2017.

\begin{tabular}{|c|c|c|c|c|c|c|}
\hline Año & $\begin{array}{c}\text { Vehículos } \\
\text { particulares }\end{array}$ & Carga liviana & Motocicletas & Autobuses & Taxis & Carga pesada \\
\hline 2011 & 694395 & 164025 & 151721 & 16997 & 13802 & 36062 \\
\hline 2012 & 718423 & 169704 & 156976 & 17582 & 14279 & 37311 \\
\hline 2013 & 746942 & 176444 & 163214 & 18275 & 14845 & 38792 \\
\hline 2014 & 785526 & 185562 & 171653 & 19212 & 15612 & 40797 \\
\hline 2015 & 830223 & 196124 & 181429 & 20298 & 16500 & 43119 \\
\hline 2016 & 872007 & 205997 & 190568 & 21312 & 17330 & 45289 \\
\hline 2017 & 904629 & 213707 & 197704 & 22105 & 17978 & 46984 \\
\hline
\end{tabular}


vehículo y para cada año modelo. Siendo seis tipos de vehículos y hasta 40 años modelo (escenario 20 I5), resultan 240 ecuaciones de curvas de retiro por construir. En la figura 5 se muestra el comportamiento de estas curvas de retiro para tres años distintos y varias clases de vehículos.

Con base en los modelos de las curvas de retiro descritas y los modelos econométricos, así como en las estimaciones de las incorporaciones, se construyen los parques vehiculares por tipo de vehículo y año modelo para los escenarios propuestos.

Escenario I: Reducir el contenido de azufre en el diesel de 400 a 100 ppm para 2015

Se analizó el impacto de la distribución y consumo de diesel con concentraciones cercanas a 100 ppm a partir de 2015. Con este propósito se establecieron dos escenarios: escenario 0 , que consiste en el estado de las emisiones contaminantes proyectadas a 2015 con diesel de 400 ppm, y el escenario I, que corresponde al estado de las emisiones contaminantes proyectadas a 2015 con diesel de 100 ppm de azufre. Los efectos de esta medida se determinaron obteniendo la diferencia neta entre las emisiones de azufre en forma de óxidos de azufre cuando se consume diesel con 400 ppm de azufre y diesel con 100 ppm, aplicando para ello el software Mobile 6. Si se analizan los resultados obtenidos (cuadro 9) se puede ver que esta medida produciría una reducción del 75\% ( 120 I ton) en las emisiones de dióxido de azufre. Esta reducción en las emisiones se sumaría al esfuerzo realizado en el año 2007, cuando el contenido de azufre en diesel se disminuyó de 3500 a 400 ppm (figura 6).

Escenario 2: Reemplazo del 100\% de unidades de autobuses $y$ taxis con edades superiores a los 15 años para 2015

Con este escenario se propone la sustitución de 7015 unidades de autobuses ( 104 que operan con gasolina y 691 I con diesel) y 6696 taxis (6137 que operan con gasolina y 559 con diesel) que tengan una edad promedio mayor o igual a I 5 años para el año 20I5. La sustitución de las unidades se plantea bajo dos posibilidades: primero un reemplazo del total de las unidades en el año 2015 o en forma alternativa, y segundo, una sustitución gradual en partes iguales en el período comprendido entre 2013-2015.
Con el propósito de evaluar el impacto de esta medida se establecieron tres escenarios: el escenario 0 , que consiste en el estado de las emisiones contaminantes proyectadas a 2015 sin la sustitución
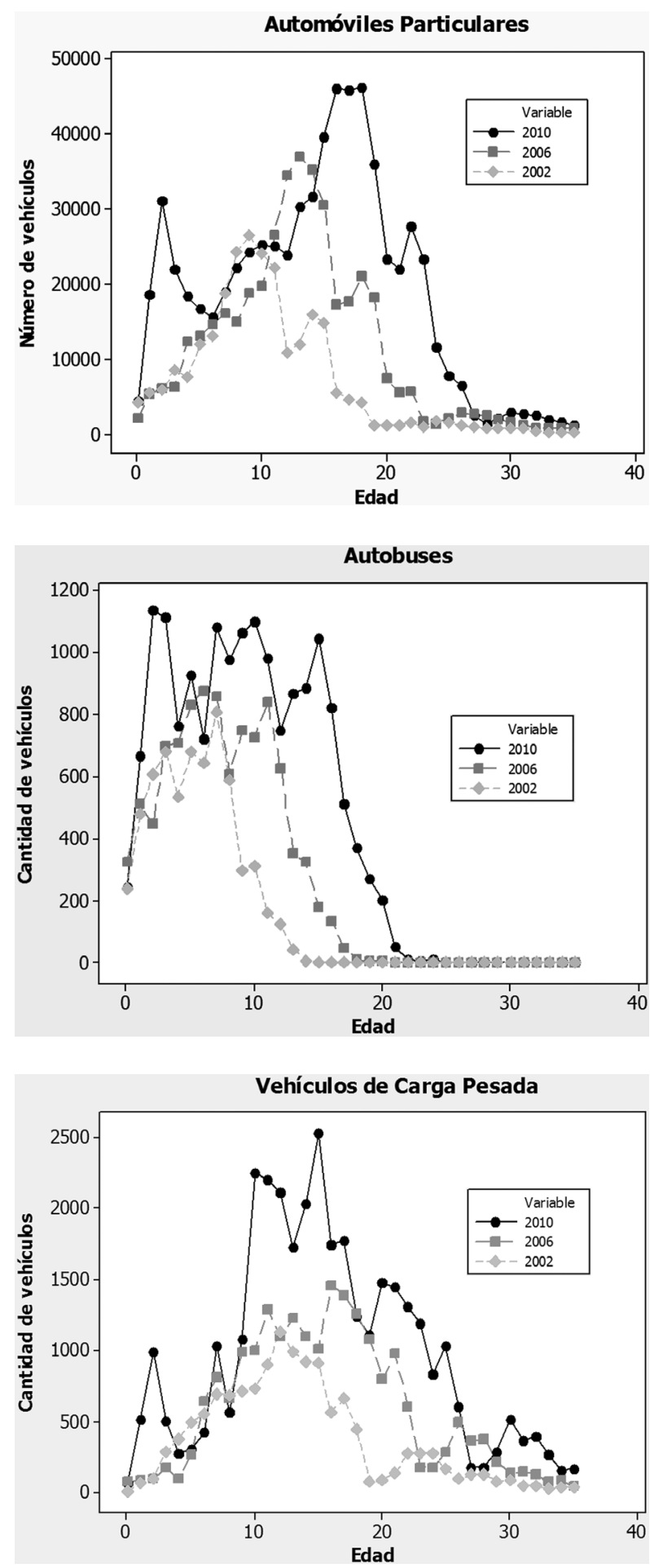

Figura 5. Curvas de retiro de vehículos en Costa Rica para tres años diferentes. 
Cuadro 9. Emisiones de $\mathrm{SO}_{2}$ (toneladas) generadas bajo los dos escenarios planteados en materia de combustibles por las unidades de la flota que operan con diesel.

\begin{tabular}{|c|c|c|c|c|c|c|}
\hline & Autobuses & Taxis & Carga pesada & Carga liviana & $\begin{array}{c}\text { Automóviles } \\
\text { particulares }\end{array}$ & Total \\
\hline Diesel con S 400 ppm & 301 & 43 & 332 & 723 & 204 & 1603 \\
\hline Diesel con S 100 ppm & 76 & 11 & 83 & 181 & 51 & 402 \\
\hline
\end{tabular}

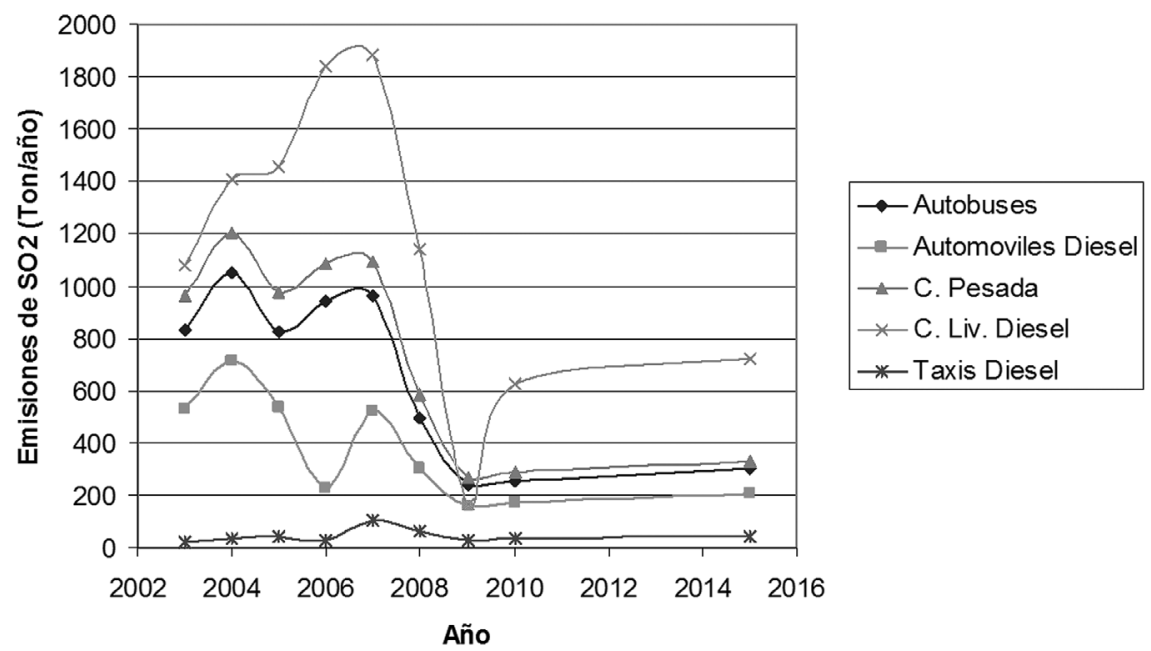

Figura 6. Comportamiento de las emisiones anuales de dióxido de azufre en Costa Rica por categoría de vehículo.

Cuadro 10. Emisiones generadas (toneladas) producto de los distintos escenarios de intervención en la flota de autobuses y taxis.

\begin{tabular}{|c|c|c|c|}
\hline & GOT & CO & NOx \\
\hline 2015 sin sustitución & 4848 & 16104 & 23249 \\
\hline $\begin{array}{c}2015 \text { con sustitución } \\
100 \% \text { en } 2015\end{array}$ & 1946 & 9243 & 13732 \\
\hline $\begin{array}{c}2015 \text { con sustitución en } \\
\text { el período 2013-2015 }\end{array}$ & 2361 & 10736 & 18397 \\
\hline
\end{tabular}

de las unidades; el escenario I, que implica el estado de las emisiones contaminantes proyectadas a 2015 con la sustitución total en ese año; y el escenario 2, que conlleva el estado de las emisiones proyectadas a 2015 pero con un sustitución anual gradual de iguales proporciones del 2013-20I5. Los efectos se calculan obteniendo la diferencia neta entre las emisiones contaminantes que dejan de emitirse al retirar las unidades de los años modelo más antiguos. Se proyectó la flota vehicular de acuerdo a las curvas de retiro para determinar las cantidades y edad de las unidades por sustituir. Los resultados obtenidos se muestran en el cuadro 10. Tal como se puede observar en dicha tabla, el escenario de realizar la sustitución del 100\% de las unidades de autobuses y taxis con edades mayores a 15 años para el 20I5, en un período de tiempo de un año, representa la mayor reducción de emisiones de contaminantes, con un $47,8 \%$ en comparación con el 35,2\% de la sustitución parcial a tres años.

La implementación de la medida en los autobuses representa un 54\% del total de la reducción de la emisión de contaminantes bajo el escenario de sustitución total del 100\% en el 2015, mientras que la contribución de los taxis corresponde a un $45 \%$ para las unidades que operan con gasolina y tan sólo un $0,7 \%$ para las de diesel. 
Escenario 3:Aplicación de medidas de restricción vehicular de un día por semana de acuerdo con el número de placa del vehículo

En el alcance de esta acción se propone el establecimiento de un sistema de restricción a la circulación orientado en dos alternativas: i) restricción de un día a la semana a todos los vehículos de la flota de acuerdo con el número de terminación de la placa, y ii) restricción de circulación de un día a la semana solo a las unidades de la flota con más de 10 años de antigüedad.

Se empleó el método econométrico para proyectar la flota vehicular para el año 2015 que dejan de circular. Las edades se proyectaron en función de las curvas específicas de retiro. Posteriormente se realizaron las correcciones correspondientes a los datos de kilómetros recorridos totales por tipo de vehículo y combustible, considerando la restricción de circulación de un día a la semana.

Tal como se puede observar en el cuadro II, el establecimiento de un sistema de restricción vehicular causaría un impacto más significativo en las emisiones de $\mathrm{PM}_{10}$ y $\mathrm{SO}_{2}$ con tasas de reducción cercanas al 22\%, en comparación con un 15\% en promedio para los contaminantes restantes. En forma adicional, los automóviles particulares y vehículos de carga liviana presentan los mayores niveles de reducción (20\%) en promedio para los contaminantes analizados al entrar en vigencia la restricción vehicular.

Al examinar los dos modelos de restricción propuesta se puede observar que no se obtiene una ventaja adicional significativa al aumentar la restricción a toda la flota, ya que, por ejemplo, en el caso de carga liviana, carga pesada y automóviles particulares el efecto es de tan solo un $2 \%$ adicional, por lo que en primera instancia se recomienda la restricción solo a las unidades con una edad mayor a 10 años al 2015.

\section{Escenario 4: Prohibición de importación de vehículos usados con más de cinco años de antigüedad a partir del año 2010}

Bajo este escenario, se evaluó el impacto de establecer controles más estrictos sobre la importación de vehículos usados, específicamente sobre su edad promedio al ingresar al país, como medida para revertir la tendencia de envejecimiento de la flota vehicular. Con este fin se plantearon dos posibilidades de restricción: cinco o diez años de antigüedad como edad máxima de los vehículos al ingresar al país.

Para realizar este análisis se utilizaron modelos econométricos ajustados y las curvas de retiro para

Cuadro I I. Estimación de las emisiones generadas (toneladas/año) por el establecimiento de una medida de restricción vehicular bajo dos escenarios distintos.

\begin{tabular}{|c|c|c|c|c|c|c|c|c|c|c|c|c|c|c|c|}
\hline $\begin{array}{l}\text { Tipo de } \\
\text { vehículo }\end{array}$ & \multicolumn{3}{|c|}{ GOT } & \multicolumn{3}{|c|}{$\mathrm{CO}$} & \multicolumn{3}{|c|}{$\mathrm{NOx}$} & \multicolumn{3}{|c|}{$P M_{10}$} & \multicolumn{3}{|c|}{$\mathrm{SO}_{2}$} \\
\hline Escenario & I & 2 & 3 & I & 2 & 3 & I & 2 & 3 & I & 2 & 3 & I & 2 & 3 \\
\hline Autobuses & $|43|$ & 1515 & | 669 & 5125 & 5417 & 5977 & 17115 & | 8091 & 19959 & 129,8 & 138,1 & 194 & 258 & 273 & 301 \\
\hline Taxis & 2726 & 2754 & 3179 & 8684 & 8840 & 10127 & 2880 & 2965 & 3290 & 39,8 & 43,4 & 47 & 41,5 & 46,2 & 49 \\
\hline Carga liviana & 6580 & 6714 & 10561 & 78056 & 79462 & 91019 & 22043 & 23262 & 25703 & 489 & 524 & 720 & 758 & 805 & 739 \\
\hline $\begin{array}{l}\text { Carga } \\
\text { pesada }\end{array}$ & 1280 & | 299 & | 493 & 6825 & 6975 & 7961 & 10989 & 11240 & 12815 & 247,9 & 256,2 & 289 & 284,4 & 291,8 & 332 \\
\hline $\begin{array}{l}\text { Automóviles } \\
\text { particulares }\end{array}$ & 54495 & 54695 & 61773 & | 4874 | & 152396 & 163037 & 45966 & 47947 & $48 \quad 86$ & 339 & 357 & 450 & 117 & 123 & 289 \\
\hline Motocicletas & 13306 & 14664 & 15516 & 50214 & 55839 & 55841 & 2339 & 2645 & 2728 & 58,1 & 65,9 & 68 & 63,6 & 71,9 & 74,1 \\
\hline
\end{tabular}

Escenario I: restricción de un día a todas las unidades de la flota.

Escenario 2: restricción de un día solo a unidades con edad mayor a 10 años.

Escenario 3: sin restricción vehicular. 
estimar la distribución de la flota vehicular por edades al año 20 I5, bajo las tres condiciones planteadas en el alcance del presente escenario. Tal como puede observarse en el cuadro 12, resulta más conveniente, en términos de reducción de emisiones, limitar la importación de vehículos con más de 5 años de antigüedad, ya que mientras bajo esta condición se genera una reducción promedio de $2,71 \%$ en las emisiones, si se restringe a 10 años el descenso es de tan solo el 1,40\% en promedio. Dentro de los contaminantes contemplados, esta medida favorece considerablemente el control de los gases orgánicos totales y los óxidos de nitrógeno mayoritariamente, con disminuciones de 2,59 y 3,39\% respectivamente. En el caso de los gases orgánicos, los principales descensos se presentan en las emisiones de las motocicletas (6,80\%), taxis (4,59\%) y vehículos de carga liviana (2,97\%), mientras que para el caso de los óxidos de nitrógeno las principales reducciones se presentan en los taxis $(5,28 \%)$ y los vehículos particulares (4,52\%).

Al analizar los resultados se puede considerar que las categorías de vehículos que más contribuyen al descenso de las emisiones al restringir la edad de ingreso a 5 años son los taxis (3,53\%), motocicletas $(3,47 \%)$, carga liviana $(2,47 \%)$ y carga pesada (2,38\%). para el año 2015, dando prioridad de sustitución a todas aquellas que tengan más de 15 años promedio de edad. Las nuevas unidades podrán ser reemplazadas por: (a) unidades que operan con gas licuado de petróleo o (b) vehículos de tecnologías más limpias. La sustitución se iniciaría en el año 2012 y finalizaría en 2014.

En el caso de los autobuses, se plantea incorporar como vehículos más limpios unidades EURO-IV. Para estimar las emisiones de estas unidades, se obtuvieron los factores de emisión de la norma oficial mexicana NOM-044-SEMARNAT-2006. La conversión de unidades se realizó considerando un rendimiento de combustible de $2,3 \mathrm{~km} / \mathrm{l}$ de acuerdo con estudios recientes (SMADF, 2006). El resto de los factores de emisión se obtuvo ponderando los resultados del modelo Mobile 6 y por balance de materia. Para los taxis se consideraron unidades TIER-2 para utilizarlas en reemplazo de los vehículos. Los factores de emisión aplicables al programa TIER-2 se presentan en el cuadro 13. El resto de los factores se obtuvo por balance de materia y del modelo Mobile 6.

En el cuadro 14 se puede observar que con el reemplazo de las unidades más antiguas por unidades que operan con GLP se obtiene en promedio una reducción del $45 \%$ en las emisiones,

Cuadro 12. Emisiones de contaminantes (toneladas/año) generadas al año 2015 bajo distintos escenarios de regulación de la importación de vehículos usados.

\begin{tabular}{|l|c|c|c|c|c|}
\hline & GOT & CO & NOx & PM $_{10}$ & $\mathrm{SO}_{2}$ \\
\hline Sin regulación a las importaciones & 94191 & 336676 & 112681 & 1767 & 1784 \\
\hline Edad máxima de ingreso 5 años & 91751 & 329104 & 108948 & 1762 & 1763 \\
\hline Edad máxima de ingreso 10 años & 93148 & 331382 & 110971 & 1765 & 1776 \\
\hline
\end{tabular}

Escenario 5: Incentivar el uso de gas licuado de petróleo o unidades de alto rendimiento en el $50 \%$ de las unidades de transporte público para el año 2015

Dentro de este escenario se plantea sustituir el 50\% de las unidades de transporte público (taxis y autobuses) que se proyecta se encuentren en operación
Cuadro 13. Factores de emisión $(\mathrm{g} / \mathrm{km})$ para taxis TIER-2.

\begin{tabular}{|c|c|c|c|c|c|}
\hline \multicolumn{3}{|c|}{ TIER 2 } & \multicolumn{2}{c|}{ Mobile 6 } \\
\hline CO & GOT & NOx & $\mathrm{PM}_{10}$ & $\mathrm{NH}_{3}$ & $\mathrm{SO}_{2}$ \\
\hline 2,610 & 0,056 & 0,043 & 0,006 & 0,0632 & 0,0042 \\
\hline
\end{tabular}


Cuadro 14. Emisiones de contaminantes (toneladas/año) generadas al año 2015 bajo distintos escenarios de renovación del $50 \%$ de las unidades destinadas al transporte público.

\begin{tabular}{|c|c|c|c|c|c|c|}
\hline \multicolumn{2}{|c|}{ Categorías } & GOT & $\mathrm{CO}$ & NOx & $\mathrm{SO}_{2}$ & $\mathrm{PM}_{10}$ \\
\hline \multirow{3}{*}{ Autobuses } & $\begin{array}{c}\text { 50\% operando con } \\
\text { GLP }\end{array}$ & 1097 & 3221 & 10266 & 142 & 76 \\
\hline & $\begin{array}{l}50 \% \text { unidades alta } \\
\text { eficiencia }\end{array}$ & 1330 & 4864 & 9402 & 153 & 84 \\
\hline & Sin sustitución & 1669 & 5977 & 19959 & 301 & 194 \\
\hline \multirow{3}{*}{ Taxis gasolina } & $\begin{array}{c}\text { 50\% operando con } \\
\text { GLP }\end{array}$ & 367 & 2420 & 938 & 2 & 6 \\
\hline & $\begin{array}{c}\text { 50\% unidades } \\
\text { TIER-2 }\end{array}$ & 240 & 3939 & 637 & 5 & 10 \\
\hline & Sin sustitución & 2901 & 9909 & 2739 & 6 & 17 \\
\hline \multirow{3}{*}{ Taxis diesel } & $\begin{array}{c}\text { 50\% operando con } \\
\text { GLP }\end{array}$ & 205 & 197 & 468 & 37 & 26 \\
\hline & $\begin{array}{l}50 \% \text { unidades alta } \\
\text { eficiencia }\end{array}$ & 204 & 200 & 458 & 43 & 30 \\
\hline & Sin sustitución & 278 & 218 & 551 & 43 & 30 \\
\hline
\end{tabular}

mayor a la registrada si la sustitución se realiza con unidades nuevas de alta tecnología, donde se disminuye tan solo un 36\%. Las reducciones de emisiones más importantes se presentan en autobuses, principalmente en $\mathrm{PM}_{10}(60,8 \%), \mathrm{SO}_{2}$ $(52,8 \%)$ y $\mathrm{NO} \times(48,6 \%)$.

El estado de las emisiones generadas por fuentes móviles en Costa Rica a mediano plazo (año 2015) considerando que no se aplicara acción alguna para cambiar las tendencias actuales de emisiones contaminantes, indica una ralentización del crecimiento de los volúmenes de emisiones contaminantes. Mientras que durante los seis años del periodo entre el año 2006 y el año 2012 las emisiones de $\mathrm{NOx}$ crecen a una tasa promedio de $2,6 \%$ anual, durante los tres años del periodo 2012-2015 las emisiones se incrementarán a una tasa promedio de 3,5\% anual, de acuerdo con las proyecciones desarrolladas en este trabajo.

\section{Bibliografía}

Adler, U. (1997). Bosch Automotive Handbook. 4 ed. Stuttgart: Robert Bosch $\mathrm{GmbH}$.

Bishop, G.A. \& Stedman, D.H. (1990). On-road carbon-monoxide emission measurement comparisons for the 1988-1989
Colorado oxy-fuels program. Environmental Science and Technology 24(6): 843-847.

Button, K. (1993). Transport, the Environment and Economic Policy. Aldershot, UK: Edward Elgar.

Cardelino, C. (1998). Daily variability of motor vehicle emissions derived from traffic counter data. Journal of the Air \& Waste Management Association 48(7): 637-645.

Gasca, J.R. (2005). Escenarios de emisiones y medidas de mitigación de gases de efecto invernadero en sectores clave (Transporte y Desechos). México: Instituto Mexicano del Petróleo.

Joumard, R., Jost, P., Hickman, J. \& Hassel, D. (1995). Hot passenger car emissions modelling as a function of instantaneous speed and acceleration. Science of the Total Environment 169(I-3): 167-174.

Kelly, N.A. \& Groblicki, P.j. (1993). Real-world emissions from a modern production vehicle driven in Los Angeles. Journal of the Air \& Waste Management Association 43( I0): I 35 I - 1357.

Ortizar, J.D. \& Willumsen, L.G. (1994). Modelling transport. 2 ed. UK: John Wiley \& Sons.

Parks, P. \& Hrunka, G. (2000). Creating a market for reduced carbon monoxide emissions from mobile sources. Environmental Science \& Policy 3, 47-54.

SMADF (2006). Pruebas de Campo de Autobuses de Tecnologías Alternativas en la Ciudad de México, Reporte Final. México: s.n. 